\title{
Depth Dependent Oxidation States in a PtRu Thin Film
}

\author{
C. Srivastava*, G.B. Thompson*, D. Reinhard**, T.J. Prosa** and D.J. Larson** \\ *University of Alabama, Department of Metallurgical \& Materials Engineering, $2017^{\text {th }}$ Ave, A129 \\ Bevill Building, Tuscaloosa, AL 35487-0202 \\ **Imago Scientific Instruments, 6300 Enterprise Lane, Suite 100, Madison, WI 53719-1193
}

The Pt-Ru alloy has received considerable attention because of its superior catalytic properties for use as the anode in automotive fuel cells. In particular, this alloy exhibits good CO tolerance. During methanol oxidation, intermediate $\mathrm{CO}$ is formed which binds strongly to Pt thereby blocking catalytic activity for pure Pt surfaces. When $\mathrm{Ru}$ is included, the $\mathrm{Ru}$ site catalyzes the oxidation of $\mathrm{CO}$ to $\mathrm{CO}_{2}$ and prevents $\mathrm{CO}$ poisoning of the Pt surface [1]. Thus, understanding how $\mathrm{Ru}$ is distributed over a Pt surface is critical. Atom probe tomography provides near atomic resolution of each element in 3-dimensions and is an ideal technique to provide this essential level of materials characterization. By being able to pin-point each atom, we can provide accurate physical descriptions of the surfaces to determine potential surface segregation effects which could enrich specific regions (free surfaces, grain boundaries, etc.) and change the catalytic response.

$\mathrm{A} \mathrm{Pt}_{55} \mathrm{Ru}_{45}$ thin film was sputter deposited onto a prefabricated sharpened Ag metal tip. The support needle provides a ready-surface for atom probe analysis. The atom probe technique requires a hemispherical shaped surface with a radius of curvature of $\sim 50-100 \mathrm{~nm}$ to enhance the electric field to allow for field evaporation from the surface using experimentally attainable voltages. The film was grown by co-continuous sputtering elemental $\mathrm{Pt}(99.95 \%)$ and $\mathrm{Ru}(99.95 \%)$ targets with a working gas of UHP Ar at 4.5 mTorr. Prior to sputtering, the base pressure was $\sim 5 \times 10^{-7}$ Torr. Postdeposition, the needle was placed in a Local Electrode Atom Probe (LEAP) [2]. The needle was cryogenically cooled to $130 \mathrm{~K}$ and field evaporated at $200 \mathrm{kHz}$ at a $1 \%$ field evaporation rate

Surprisingly, the film exhibited a significant degree of oxidation of both metal species as a function of film depth, as seen in Fig. 1. Since this film was not annealed or exposed to an intentional oxidizing environment, it is suspected that background oxygen within the chamber was the oxygen source. Additional specimens, grown with different background pressures, are under investigation. In this particular specimen, we observe a preferential formation of dominate oxidation states as a function of depth. PtO was found to be near the surface of the film while a $\mathrm{RuO}$ was dominate under the surface, as shown in compositional profile of Fig. 1. Though $\mathrm{PtO}_{2}$ and $\mathrm{RuO}_{2}$ are thermodynamically more stable than $\mathrm{PtO}$ or $\mathrm{RuO}$, observed field evaporation constituents are dominated by the latter. This suggests that either insufficient oxygen and/or time are present to allow the stable oxide to form. At all depths, minimal Ru was present demonstrating its strong tendency to oxidize. In the subsurface microstructure, we observed Pt-rich nano-clusters, shown in Fig. 2 as isoconcentration surfaces of 52.5at.\%. It is commonly observed that when metals are grown on an insulator surface, they can 'ball-up' (dewet) to minimize the interfacial energy associated with contact [3]. We suspect that the high interfacial energy between Pt-metal and Ru-oxide caused the Pt to form this unique morphology. As the deposition continued, the Pt-based clusters were encapsulated within the film. The dominant Pt oxide found on the surface formed when the Pt metal was exposed to the atmosphere after being retrieved from the vacuum chamber. 
References:

[1] L Gao, HL Huang, and C Korzeniewski, Electrochimica Acta 49(8) (2004) 1281.

[2] T.F. Kelly and D.J. Larson, Mat. Char. 44 (2000) 59.

[3] C.T. Campbell, Surface Science 27 (1997) 1.

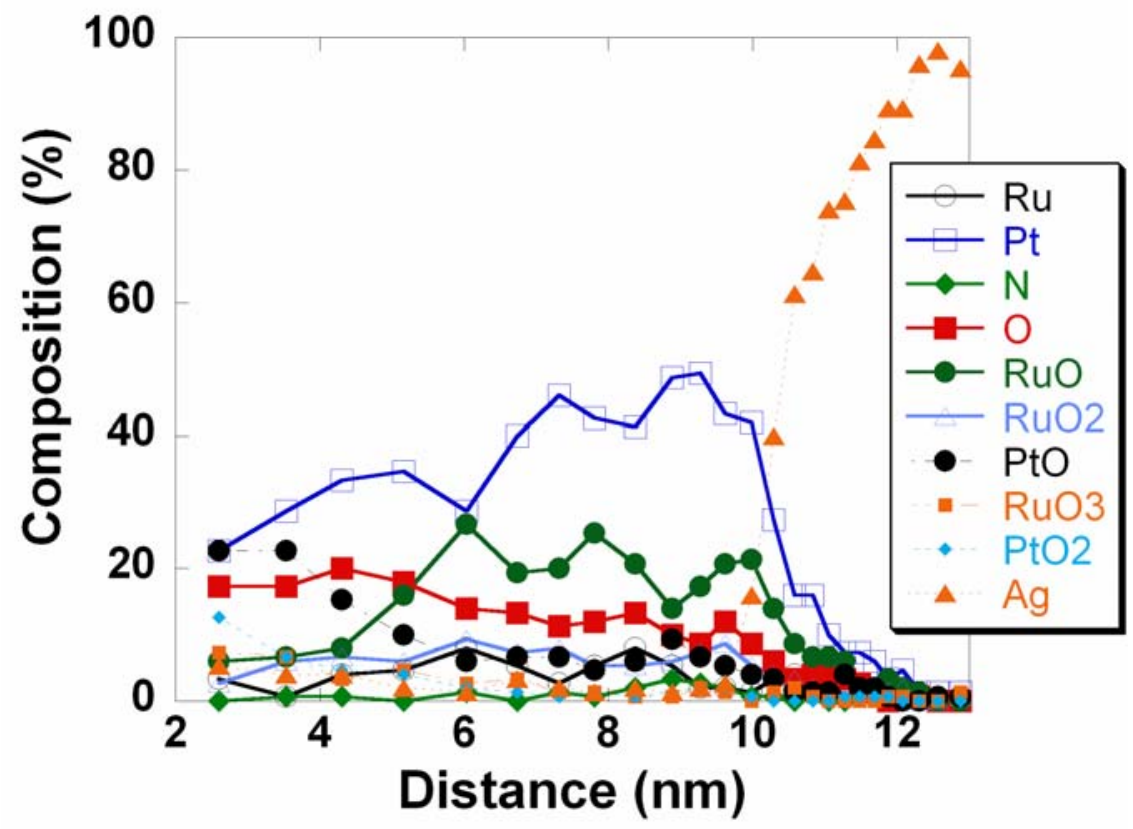

Fig.1: Composition profile of a PtRu thin film. Note the oxidation depth dependence between Pt and $\mathrm{Ru}$.

(a)

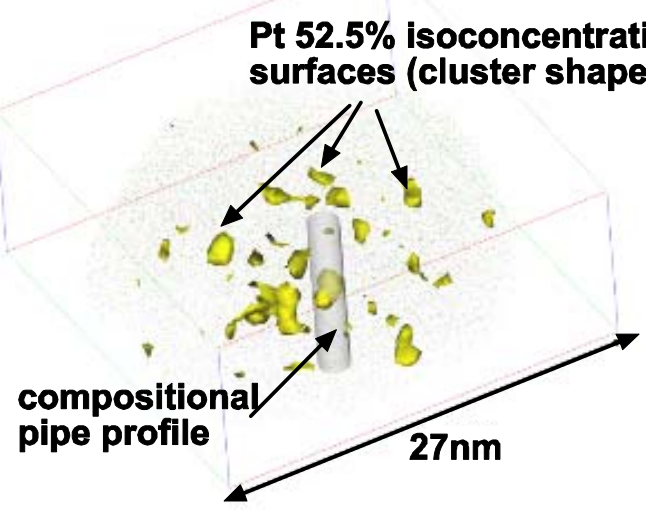

(b)

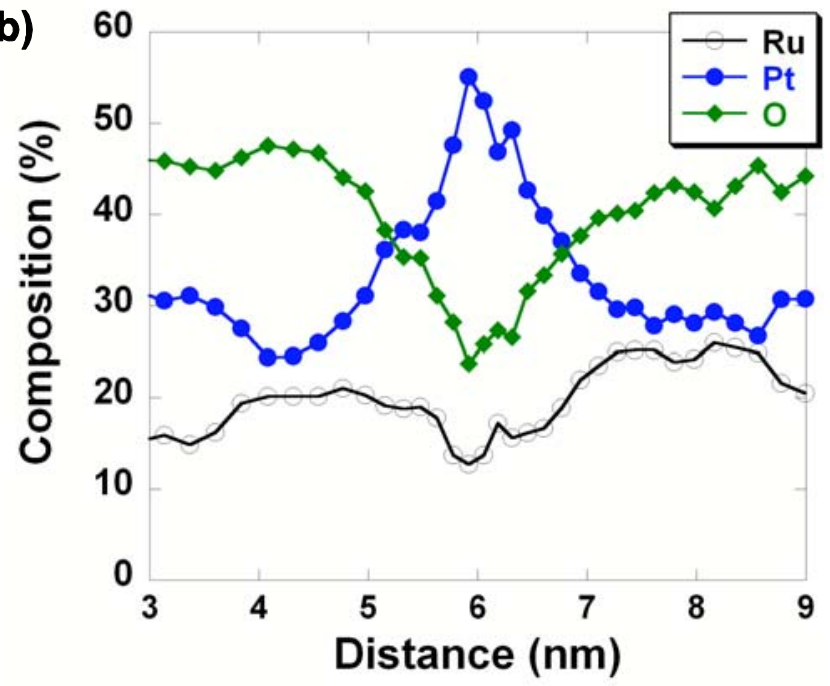

Fig. 2: (a) Isoconcentration of sub-surface Pt-rich nanoclusters. (b) The composition through a cluster. 